\title{
Neurofibromatosis tipo 1 (NF1) o enfermedad de Von Recklinghausen
}

\author{
Sergio A. Arbeláez Eslait'; Koretta Esmeral Atehortúa²; Sebastián Peluffo Vergara³
}

\section{RESUMEN}

La neurofibromatosis tipo 1 (NF1) es una enfermedad genética autosómica dominante y de compromiso multisistémico. Los signos y síntomas varían de acuerdo con la variedad fenotípica presentada. Afecta a 1 de cada 5000 individuos. El diagnóstico es confirmado por estudio citogenético.

Se discute el caso de un paciente de 70 años, que desde la niñez presenta lesiones nodulares de tamaño y forma variable, de localización generalizada en toda la extensión de la superficie corporal.

PALABRAS CLAVE: neurofibromatosis; neurofibroma; manchas café con leche; mutación.

\section{NEUROFIBROMATOSIS TYPE 1 (NF1) OR VON RECKLINGHAUSEN DISEASE}

\section{SUMMARY}

Neurofibromatosis type 1 is an autosomal dominant disorder, with multisystemic involvement. Signs and symptoms are variable according to the phenotypic variety presented. It affects 1 in 5,00o individuals. The diagnosis is confirmed by the cytogenetic study.

The case of a 70-years-old patient is discussed, with a history of nodular lesions of varying size and shape since childhood, with generalized location on the entire body surface.

KEY WORDS: Neurofibromatosis; Neurofibroma; Café au lait spots; Mutation.

1. Médico interno, Hospital Universidad del Norte, Soledad, Atlántico, Colombia. Médico interno, Hospital Civil "Fray Antonio Alcalde”, Guadalajara, Jalisco, México. ORCID https://orcid.org/oooo-ooo3-2421-6861

2. Médico interno, Hospital Universidad del Norte, Soledad, Atlántico, Colombia. Médico interno, Hospital Civil "Fray Antonio Alcalde”, Guadalajara, Jalisco, México. ORCID https://orcid.org/oooo-ooo1-9596-8046

3. Médico interno, Hospital Universidad del Norte, Soledad, Atlántico, Colombia. Médico interno, Hospital Civil "Fray Antonio Alcalde”, Guadalajara, Jalisco, México. ORCID https://orcid.org/oooo-ooo3-2402-9476

Correspondencia: Koretta Esmeral Atehortúa; email: kari_smerald@hotmail.com

Recibido: 11/10/19; aceptado: 29/04/20

Cómo citar: Arbeláez-Eslait SA, Atehortúa KE, Peluffo-Vergara S. Neurofibromatosis tipo 1 (NF1) o enfermedad de Von Recklinghausen. Rev Asoc Colomb Dermatol. 2020;28(1):82-87. DOI: https://doi.org/10.29176/2590843X.1493

Financiación: ninguna, conflictos de interés: ninguno 


\section{INTRODUCCIÓN}

La neurofibromatosis (NF) es una enfermedad genética de tipo autosómico dominante, de afectación multisistémica y presentación clínica variable, ya que se conocen 9 fenotipos de la enfermedad ${ }^{(1-3)}$.

La prevalencia de la NF1 o enfermedad de Von Recklinghausen es de 1 en 50.000 nacidos vivos y su edad de aparición es variable, con diagnóstico en la niñez en su mayoría. La incidencia es de 1 en 3000 nacidos vivos, con expresividad variable y sin preferencia de género ${ }^{(4)}$.

Está relacionada con la mutación del gen $\mathrm{NF} 1$, en el locus 17q11,2, que codifica para una proteína denominada neurofibromina, que actúa como supresor tumoral y, a su vez, inhibe la estimulación del crecimiento y proliferación celular ${ }^{(5)}$.

Las manifestaciones clínicas aumentan con la edad y pueden variar en número, forma e intensidad de paciente a paciente.

El diagnóstico de $\mathrm{NF} 1$ es principalmente clínico y se confirma con estudios de extensión citogenética, que muestran la mutación del gen antes mencionado ${ }^{(6)}$.
A continuación, se expone un reporte de caso de un paciente de 70 años, que desde la niñez presenta lesiones nodulares de tamaño y forma variable, de localización generalizada en toda la extensión de la superficie corporal.

\section{PRESENTACIÓN DEL CASO}

Se presenta el caso de un paciente masculino de 70 años, que desde la niñez presenta lesiones de tipo nodular de tamaño variable, localizadas de forma difusa en rostro, cuello, tronco, dorso y extremidades, de coloración similar a su fototipo de piel, que posteriormente se generalizaron, comprometiendo casi la totalidad de la superficie corporal.

Paciente previamente sano, sin antecedentes médicos personales de importancia. Antecedentes de neurofibromatosis tipo NF1 en su padre.

En el examen físico se evidencian múltiples nódulos rosados pediculados, con base vascularizada, de tamaño variable, localizados en áreas extensas del tronco y la cara (figura 1).



Figura 1. Imagen del examen físico al ingreso. A) Múltiples nódulos rosados localizados de forma generalizada en el dorso del paciente. B) Múltiples nódulos rosados localizados en forma difusa en el rostro del paciente. C) Múltiples nódulos rosados localizados en el abdomen, el tórax y el cuello. 
En estudios de extensión se presentó cariotipo 46,XY, con mutación del gen NF1 en el locus 17q11,2, que codifica para la proteína neurofibromina. Estudios de audiometría reportaron preservación de la audición bilateral. Estudios imagenológicos de sistema cardiovascular, nervioso y renal fueron normales.

De acuerdo con los hallazgos, el paciente fue diagnosticado con neurofibromatosis de tipo NF1.

\section{DISCUSIÓN}

Las neurofibromatosis son un grupo de patologías que incluyen tres entidades heterogéneas: neurofibromatosis tipo 1 (NF1), neurofibromatosis tipo $2\left(\mathrm{NF}_{2}\right)$ y schwannomatosis $^{(1)}$ (tabla 1).

La NF1 es una enfermedad genética, autosómica dominante, ocasionada por la mutación en el gen NF1, en el locus 17q11,2. Este gen codifica para una proteína supresora tumoral, denominada neurofibromina, que es similar a las proteínas activadoras del Ras-GTPasa; su función es la inhibición de la proliferación y crecimiento celular ${ }^{(2)}$.

Tabla 1. Tipos de neurofibromatosis
Se estima que la prevalencia de la NF1 es de 1 en 50.000 nacidos vivos, y la incidencia es de 1 en 3000. La NF1 tiene penetrancia del $100 \%$ y expresividad variable ${ }^{(2-4)}$.

Existen diferentes tipos de presentación clínica, que incluyen lesiones dermatológicas diferentes y afecciones sistémicas en distintos niveles. Principalmente, compromete al sistema nervioso central y periférico, incluidos el sistema cardiovascular, gastrointestinal y alteraciones musculoesqueléticas (tabla 2) ${ }^{(1,2)}$.

El diagnóstico se hace frecuentemente en la niñez, por la aparición congénita o temprana de las lesiones, sumado a los antecedentes familiares. El Instituto Nacional de Salud de Estados Unidos (NHI), en 1982, realizó un consenso donde se establecieron los criterios clínicos para el diagnóstico de NF. Se deben cumplir con 2 o más criterios para la confirmación del diagnóstico (tabla 3) ${ }^{(6,7)}$.

Clínicamente, se distinguen tres tipos de presentación de los neurofibromas, que varían de acuerdo con el lugar de aparición y la característica de la lesión (tabla 4) ${ }^{(8)}$.

\begin{tabular}{|c|c|c|c|}
\hline Tipo & Gen & Fenotipo & Neoplasia asociada \\
\hline \multirow{3}{*}{ NF1 } & \multirow{3}{*}{ NF1 } & $\begin{array}{l}\text { Déficit de atención y } \\
\text { aprendizaje }\end{array}$ & Glioma óptico \\
\hline & & $\begin{array}{l}\text { Displasias óseas y } \\
\text { alteraciones de la } \\
\text { pigmentación cutánea }\end{array}$ & Neurofibroma cutáneo \\
\hline & & & Neurofibroma plexiforme \\
\hline NF2 & $\mathrm{NF} 2$ & $\begin{array}{l}\text { Hamartomas retinianos y } \\
\text { cataratas juveniles }\end{array}$ & $\begin{array}{l}\text { Schwannomas } \\
\text { vestibulares }\end{array}$ \\
\hline \multirow{3}{*}{ Schwannomatosis } & LZTR1 & \multirow{3}{*}{ Ninguno } & \multirow{3}{*}{ Schwannomas } \\
\hline & & & \\
\hline & SMARCB1 & & \\
\hline
\end{tabular}

Fuente: construida por los autores. 
Tabla 2. Clasificación de Riccardi de la neurofibromatosis

$\begin{array}{ll}\text { Tipo } & \text { Característica } \\ \text { NF1 } & \text { Manchas café con leche y neurofibromas } \\ \text { NF2 } & \text { Schwannomas acústicos bilaterales } \\ \text { NF3 } & \text { Neurofibromas y algunas manchas café con leche } \\ \text { NF4 } & \text { Variable, no cumplen con las características de otro } \\ \text { sF5 } & \text { subtipo } \\ \text { NF6 } & \text { Signos limitados a un segmento } \\ \text { NF7 } & \text { Manchas café con leche } \\ \text { NF8 } & \text { Aparición de signos después de los } 30 \text { años. No } \\ \text { presentan nódulos, ni manchas café con leche }\end{array}$

Fuente: construida por los autores.

Tabla 3. Criterios diagnósticos de neurofibromatosis tipo 1

\begin{tabular}{|c|c|}
\hline Criterio & Características de la lesión \\
\hline I & $\begin{array}{l}60 \text { más manchas café con leche: } \\
->0,5 \mathrm{~cm} \text { en edad prepuberal } \\
->1,5 \mathrm{~cm} \text { en edad pospuberal }\end{array}$ \\
\hline II & $\begin{array}{l}\text { - } 20 \text { más neurofibromas de cualquier tipo } \\
\text { - } 10 \text { más neurofibromas plexiformes }\end{array}$ \\
\hline III & Efélides inguinales o axilares \\
\hline IV & Gliomas ópticos \\
\hline V & 20 más nódulos de Lisch \\
\hline VI & $\begin{array}{l}\text { Lesión ósea distintiva: } \\
\text { - Displasia de hueso esfenoides } \\
\text { - Displasia o adelgazamiento de la corteza de } \\
\text { huesos largos }\end{array}$ \\
\hline VII & $\begin{array}{l}\text { Pariente en primer grado con neurofibromatosis } \\
\text { tipo } 1 \text { (NF1) }\end{array}$ \\
\hline
\end{tabular}

Fuente: construida por los autores. 
Tabla 4. Clasificación de los neurofibromas de acuerdo con la manifestación cutánea

$\begin{array}{ll}\text { Tipo } & \text { Característica } \\ \text { Cutáneo } & \text { Blandos y superficiales } \\ \text { Subcutáneo } & \text { Dolorosos y con afección nerviosa periférica } \\ \text { Plexiforme } & \begin{array}{l}\text { Afectación profunda, que incluye diferentes tipos de } \\ \text { tejidos. Existe aumento del pliegue cutáneo, que } \\ \text { hace que la piel se repliegue sobre sí misma }\end{array}\end{array}$

Fuente: construida por los autores.

Los neurofibromas característicos de la NF1 tienen variaciones histológicas tales como: neurofibromas intradérmicos benignos, que son los más frecuentes, aproximadamente, un 95\% de los casos; neurofibromas nodulares, que afectan los nervios periféricos y no infiltran los tejidos circundantes, aunque pueden generar un fenómeno de compresión por su gran tamaño; y, por último, neurofibromas plexiformes, que habitualmente son congénitos y se presentan en apro-



Cerca del 50\% de los pacientes desarrollan, concomitantemente a las manifestaciones cutáneas, alteraciones tales como: trastorno de hiperactividad y déficit de atención, alteraciones en el aprendizaje, trastornos del espectro autista, cefaleas y retraso mental y pondoestatural ${ }^{(9)}$.

Existen reportes de casos de ruptura de aneurismas en arterias de pequeño y mediano calibre, en localizaciones no habituales, que están relacionados con el diagnóstico tardío de NF1. La embolización arterial transcatéter ha sido el tratamiento útil en estos casos (10).

En este tipo de complicaciones, que ponen en riesgo la vida de los pacientes, radica la importancia de realizar un diagnóstico oportuno en la niñez, cuando las manifestaciones cutáneas se hacen evidentes.

El tratamiento de la NF1 está dirigido según la afección del paciente. Se tienen en cuenta los siguientes criterios: localización de la lesión, compromiso de la lesión (desfiguración o efecto compresivo), limitación de la funcionalidad y velocidad de crecimiento de la lesión. Puede variar desde observación y seguimiento clínico hasta intervenciones quirúrgicas para escisión parcial o total de la lesión ${ }^{(5,6,8)}$.

Estudios recientes en pacientes con NF1 han demostrado que el uso de selumetinib, un inhibidor de la proteína MEK (parte de la vía de señalización RAS), tiene un efecto regulador sobre el crecimiento y la proliferación de los neurofibromas. Su uso aplicado en pacientes con la variante más agresiva y rápidamente progresiva de la NF1, los neurofibromas plexiformes, ha evidenciado una reducción drástica en el tamaño de los tumores, lo que permite que la extirpación quirúrgica sea eficaz y con menor probabilidad de recidivas y complicaciones ${ }^{(10,11)}$.

\section{CONCLUSIONES}

Se presentó el reporte de caso de un paciente con NF1, que es una entidad neurocutánea poco frecuente, secundaria a la mutación del gen NF1 en el cromosoma 17 .

Es relevante realizar el diagnóstico temprano de las enfermedades con manifestaciones cutáneas tan evidentes, ya que se podrían evitar complicaciones cutáneas y extracutáneas de la enfermedad o, como en el caso en mención, la larga evolución de la enfermedad hace que el manejo de esta sea más complejo.

Se debe conocer las manifestaciones clínicas de los 9 subtipos de NF, debido a que, por su variabilidad feno- 


\section{Puntos clave}

- La NF1 es una patología neurocutánea poco frecuente, secundaria a la mutación del gen NF1 en el cromosoma 17.

- El Diagnóstico oportuno en etapas iniciales de la enfermedad, evita la aparición de complicaciones cutáneas y extracutáneas de la enfermedad.

- $\quad$ Existen 9 subtipos de NF, con variabilidad fenotípica, que podría que el médico diagnostique de forma más tardía la aparición de la enfermedad.

- El diagnóstico se hace por medio de los hallazgos clínicos y se confirrma con la realización de estudios citogenéticos.

- Se recomienda hacer seguimiento de los pacientes, para evaluar la aparición de nuevas lesiones y evitar la ulceración de las previas.

típica, podría llegar a confundirse con enfermedades que cursen con los signos típicos, tales como las manchas café con leche y la presencia de nódulos pediculados.

El diagnóstico se hace por medio de los hallazgos clínicos y se confirma con la realización de estudios citogenéticos.

Se recomienda hacer seguimiento de los pacientes, para evaluar la aparición de nuevas lesiones y evitar la ulceración de las previas.

\section{REFERENCIAS}

1. Cimino PJ, Gutmann DH. Neurofibromatosis type 1. Handb Clin Neurol. 2018;148:799-811. doi: 10.1016/b978-0-444-64076-5.00051-x.

2. Fitzpatrick TB. Neurofibromatosis. En: Color Atlas and Synopsis of Clinical Dermatology. New York, NY: McGraw-Hill; 1997. p. 458-62.

3. Riccardi VM. Neurofibromatosis: Clinical Heterogeneity. Curr Probl Cancer. 1982;7(2):1-34. doi: 10.1016/s0147-0272(82)80016-0.

4. Campollo Rodríguez I, Rodríguez Rojas JL, Limache Yaringaño LM. Neurofibromatosis segmentaria: presentación de un caso. Rev Arch Medico Camagüey. 2011;15(6):1020-7. Disponible en: https://bit.ly/2AVSOkK.

5. Suárez Fernández R, Trasobares L, Medina S, García Rodríguez M. Neurofibromatosis. Med Integr. 2001;38(2):64-8. Disponible en: https://bit.ly/2yrC18a.
6. Neurofibromatosis. Conference Statement. National Institutes of Health (NIH) Consensus Development. Arch Neurol. 1988;45(5):172-8.

7. Celis-Amórtegui LM, Romero-Hernández CA, Hernández-Gordillo LM, Botero-Hernández JA. Neurofibromatosis de Von Recklinghausen: presentación de un caso de neurofibroma vaginal y revisión de la literatura. Rev Colomb Obstet Ginecol. 2012;63(2):163-7. Disponible en: https://bit.ly/3eebTgl.

8. Gámez O, Correa M Rodríguez M, Miyares A, Romero D. Neurofibromatosis de tipo 1 en un adulto joven. Medisan. 2014;18(4):582. Disponible en: https://bit.ly/3gfAX8z.

9. Kokkinou E, Roka K, Alexopoulos A, Tsina E, Nikas I, Krallis P, Thanopoulou I, Nasi L, Makrygianni E, Tsoutsou E, Kosma K, Tsipi M, et al. Development of a multidisciplinary clinic of neurofibromatosis type 1 and other neurocutaneous disorders in Greece. A 3-year experience. Postgrad Med. 2019;131(7):445-52. doi.org/10.1080/00325481.2019.1659708.

10. Miyamoto K, Nakamura M, Suzuki K, Sasaki J, Dohi K, Hayashi M. Diagnosis of neurofibromatosis type 1 after rupture of aneurysm and consequent fatal hemothorax. Am J Emerg Med. 2020;So735-6757(20)30232-1. doi.org/10.1016/j.ajem.2020.04.004.

11. Duque FL, Ramírez OA. Neurofibromatosis: reporte de un caso. Rev Fac Odontol Univ Antioq. 2010;21(2):218-25. Disponible en: https://bit.ly/3cZLeDH. 